\title{
Quantum quench for the biaxial spin system
}

\author{
A.A. Zvyagin \\ B. Verkin Institute for Low Temperature Physics and Engineering of the National Academy of Sciences of Ukraine \\ 47 Nauky Ave., Kharkiv 61103, Ukraine \\ V.N. Karazin Kharkiv National University, 4 Svobody sq., Kharkiv 61002, Ukraine \\ Max-Planck-Institut für Physik komplexer Systeme, 38 Nöthnitzer Str., D-01187, Dresden, Germany \\ E-mail: zvyagin@ilt.kharkov.ua
}

Received May 14, 2018, published online September 26, 2018

\begin{abstract}
Dynamical non-equilibrium effects after a quantum quench in a quantum spin system, which permits exact analytical solution in both closed and open cases, are studied. The exact analytic results are obtained for the biaxial paramagnet, both for the "easy axis"- and the "easy-plane"-like situations, and for the field directed along both principal axes of the system. Quantum quench of the external magnetic field produces a nonlinear response. For the closed system the average magnetic moment oscillates with time and with the final value of the external field. Such oscillations exist also for the open system, connected to the bath, in the dynamical regime. For the steady-state regime in the open case the oscillations are damped. Non-equilibrium effects yield specific hysteresis phenomena in the considered single spin system.
\end{abstract}

Keywords: quantum quench, biaxial magnetic anisotropy, dynamical hysteresis.

\section{Introduction}

Quantum systems out of equilibrium, e.g., after abrupt changes of their parameters, are basically not susceptible to general principles of equilibrium systems [1]. This is why, studies of non-equilibrium dynamics of quantum models are necessary for the fundamental understanding of how mechanics emerges under the unitary time evolution. The time evolution of quantum averages depends on the initial state through the values of a large number of parameters of the quantum system. It disagrees with the standard ensembles of statistical mechanics which use few conserved values of the dynamical system and usually describe the behavior after relaxation. Theoretical studies of dynamical characteristics of many-body quantum systems are more difficult than of their static counterparts, because all eigenstates contribute to dynamics, and there is no possibility to limit the consideration by the low-energy eigenstates, as, e.g., in low-temperature thermodynamics. Since the dynamics of a quantum system typically involve many excited eigenstates, with a non-thermal distribution, the time evolution of such a system provides an unique way for investigation of non-equilibrium quantum statistical mechanics. Last decade such new subjects like quantum quenches, thermalization, pre-thermalization, equilibration, generalized Gibbs ensemble, etc. are among the most attractive topics of investigation in modern quantum physics. Abrupt changes of some parameters, i.e., quantum quenches, in which the system is prepared in an eigenstate of the initial Hamiltonian and its time evolution driven by the final Hamiltonian, lead to such a unitary time evolution, and the final (long time) state strongly depends on the type of the system. Their studies can provide the information of how fast correlations spread in quantum systems, whether averages can decay to some time-independent values, and which parameters can govern those processes. The study of the non-equilibrium dynamics of quantum coherence is very important for the modern theory of quantum computation, where namely abrupt changes (gates) are used to govern the behavior of ensembles of qubits [2]. On the other hand, the study of sudden changes is very important in the context of experiments on ultracold gases [3], ultrafast (e.g., THz) pulses [4] realized in solids [5], or high magnetic field experiments in pulse fields [6,7]. For ultracold gases, for instance, the coherence is maintained for much longer times than for usual condensed matter, and the time evolution of a quantum system after abrupt changes has become a realistic concept. The analysis of nonlinear quantum dynamics of isolated spins or small particles in the mean field approximation) was performed, e.g., in [8]. Nonlinear quan- 
tum dynamics of spins $S=1$ under action of short laser pulses has been studied in [9], see also [10] (it was shown there that such a dynamics can be totally longitudinal, i.e., with the evolution of the average value of the spin, and average values of quadrupole variables).

The new field of technology, molecular spintronics, combines the approaches and the advantages of spintronics and molecular electronics. The main issue of the molecular spintronics is the creation of small devices using one or several magnetic molecules. Single molecule magnets or single atom magnets can be used there. In such systems the magnetic relaxation time is very long at low temperatures [11]. Their single- or few-particle nature yields quantum effects of their static and dynamic magnetic properties [12]. The interest to single molecular magnets is caused by a small number of degrees of freedom (due to absent exchange between spins). Contrary, the spin-orbit coupling for a single spin together with the crystalline electric field of nonmagnetic ligands governs the magnetic properties of the system, yielding local spin symmetries.

In this study we consider the non-equilibrium dynamics of a simple quantum mechanical system, the paramagnetic quantum spin, which has the biaxial magnetic anisotropy in the external magnetic field. The advantage of the considered model is its solvability: The characteristics of the model after the quantum quench is written explicitly, in the closed form. The results are obtained for the "easy axis"like and for the "easy-plane"-like main magnetic anisotropy with the weak biaxial anisotropy, for the field directed along the axis of the largest and the weakest magnetic anisotropy. We show that for the closed system the quantum quench produces oscillations of the average magnetic moment. Those oscillations persist with time and with the value of the magnitude of the quantum quench with respect to the value, determined by the parameters of the system and the values of the initial and the final values of the field. For the open system, which exchanges the energy with the bath, such oscillations persist in the dynamical regime, for small enough time values. For large values of time, in the steady-state regime, the relaxation "smears out" the oscillations. We show that the dependence of the steady-state average magnetic moment on the values of the initial value of the field and the final one are very different from the field dependence of the same system in the stationary regime. Also, we show that the behavior of the system for switching on and off the field is also very different.

\section{The Hamiltonian}

Consider the Hamiltonian of the spin $S$ with the biaxial magnetic anisotropy in the external magnetic field

$$
\mathcal{H}=-H S_{z}+D S_{z}^{2}+E S_{x}^{2}
$$

where $S_{x, z}$ are the operators of projections of the spin, $D$ and $E$ are the magnetic anisotropy parameters, and $H$ is the external magnetic field (we use units in which the Bohr magneton and the effective $g$-factors are equal to unity).

In the representation with the diagonal $z$-component for $S=1$ we can write the Hamiltonian as

$$
\begin{gathered}
\mathcal{H}_{1}=\left(\begin{array}{ccc}
-H+D+\frac{E}{2} & \frac{E}{2} & 0 \\
\frac{E}{2} & H+D+\frac{E}{2} & 0 \\
0 & 0 & E
\end{array}\right)= \\
=\left(D+\frac{E}{2}\right) \hat{I}+\frac{1}{2}\left(\begin{array}{ccc}
-2 H & E & 0 \\
E & 2 H & 0 \\
0 & 0 & -2 D+E
\end{array}\right),
\end{gathered}
$$

where $\hat{I}$ is the unity matrix. We see that the magnetic field affects only the $2 \times 2$ subspace (with $S_{z}= \pm 1$ ). In that subspace (i.e., in the effective $2 \times 2$ matrix representation) the effective Hamiltonian can be written as

$$
\mathcal{H}_{\sigma}=-H \sigma_{z}+\frac{E}{2} \sigma_{x}
$$

The density matrix $\rho=\exp (-\mathcal{H} / T) / \operatorname{Tr}[\exp (\mathcal{H} / T)]$, where $T$ is the temperature (we use units in which the Boltzmann constant is unity), in this representation is

$$
\begin{aligned}
\rho_{\sigma} & =\left[2 \sinh \frac{\varepsilon}{T}+\exp \frac{2 D-E}{2 T}\right]^{-1} \times \\
& \times\left(\hat{I}_{\sigma^{\prime}} \cosh \frac{\varepsilon}{T}+\sigma_{z}^{\prime} \sinh \frac{\varepsilon}{T}\right),
\end{aligned}
$$

where $\hat{I}_{\sigma}$ is the unity matrix in the $2 \times 2$ subspace, and $\sigma_{z}^{\prime}=(H / \varepsilon) \sigma_{z}+(E / 2 \varepsilon) \sigma_{x}$, with

$$
\varepsilon=\frac{\sqrt{4 H^{2}+E^{2}}}{2}
$$

For the spin $S=3 / 2$ case we can write the expression for the Hamiltonian in the diagonal in $S_{z}$ representation

$$
\begin{gathered}
\mathcal{H}_{3 / 2}=\frac{3 E+D}{4} \hat{I}+\frac{1}{2} \times \\
\times\left(\begin{array}{cccc}
-3 H+4 D & \sqrt{3} E & 0 & 0 \\
\sqrt{3} E & H+E & 0 & 0 \\
0 & 0 & -H+E & \sqrt{3} E \\
0 & 0 & \sqrt{3} E & 3 H+4 D
\end{array}\right) .
\end{gathered}
$$

One can see that the magnetic field acts independently in two $2 \times 2$ subspaces (for $S_{z}=3 / 2,-1 / 2$ and $S_{z}=-3 / 2,1 / 2$, respectively). In the effective $2 \times 2 \otimes 2 \times 2$ (let us denote it as $\sigma_{1}-\sigma_{2}$ ) representation for those two subspaces the effective Hamiltonians can be written as 


$$
\mathcal{H}_{\sigma_{1,2}}=\frac{1}{2}\left[\mp \hat{I}_{1,2} H \pm \sigma_{z 1,2}(2 D-E \mp 2 H)+\sigma_{x 1,2} \sqrt{3} E\right] .
$$

The density matrix in this representation can be written as

$$
\begin{gathered}
\rho_{\sigma_{1}, \sigma_{2}}=\left[2 \exp \frac{H}{2 T} \cosh \frac{\varepsilon_{1}}{T}+2 \exp \frac{-H}{2 T} \cosh \frac{\varepsilon_{2}}{T}\right]^{-1} \times \\
\times\left[\hat{I}_{1} \exp \frac{H}{2 T} \cosh \frac{\varepsilon_{1}}{T}+\sigma_{z 1}^{\prime} \exp \frac{H}{2 T} \sinh \frac{\varepsilon_{1}}{T}+\right. \\
\left.+\hat{I}_{2} \exp \frac{-H}{2 T} \cosh \frac{\varepsilon_{2}}{T}+\sigma_{z 2}^{\prime} \exp \frac{-H}{2 T} \sinh \frac{\varepsilon_{2}}{T}\right],
\end{gathered}
$$

where $\hat{I}_{1,2}$ are the unity matrices in those two $2 \times 2$ subspaces, and $\sigma_{z 1,2}^{\prime}=\left[(H \mp D \pm E / 2) / \varepsilon_{1,2}\right] \sigma_{z 1,2}+\left(\sqrt{3} E / 2 \varepsilon_{1,2}\right) \sigma_{x 1,2}$ with

$$
\varepsilon_{1,2}=\frac{\sqrt{3 E^{2}+(2 D-E \mp 2 H)^{2}}}{2} .
$$

\section{Static characteristics}

Then the quantum mechanical average value of the projection of the magnetic moment along $z$ direction is calculated as $M_{z}=\operatorname{Tr}\left(S_{z} \rho\right)$. We can calculate that value using the effective $2 \times 2$ representations written above. For the spin $S=1$ we obtain (using $\rho=\rho_{\sigma}$ )

$$
M_{z 0}=\frac{H}{\varepsilon} \frac{2 \sinh (\varepsilon / T)}{2 \cosh (\varepsilon / T)+\exp [(2 D-E) / 2 T]} .
$$

It can be compared with the approximate expression [13]

$$
\langle S\rangle \approx \frac{H}{\sqrt{H^{2}+K^{2}}},
$$

valid at low temperatures (here $K$ is the anisotropy constant in the basis plane). One can see that for the "easy axis"-like case for $S=1$ the approximate expression is reminiscent to the exact one. On the other hand, for the "easy-plane" case the situation is different.

For the spin $S=3 / 2$ we get (using $\rho=\rho_{\sigma_{1}, \sigma 2}$ )

$$
\begin{gathered}
M_{z 0}=\left[\exp (H / 2 T) \cosh \left(\varepsilon_{1} / T\right)+\right. \\
\left.+\exp (-H / 2 T) \cosh \left(\varepsilon_{2} / 2 T\right)\right]^{-1} \times \\
\times\left(\operatorname { e x p } ( H / 2 T ) \left[\frac{(H-D+E / 2)}{\varepsilon_{1}} \sinh \left(\varepsilon_{1} / T\right)+\right.\right. \\
\left.+\frac{\cosh \left(\varepsilon_{1} / 2 T\right)}{2}\right]+\exp (-H / 2 T)\left[\frac{(H+D-E / 2)}{\varepsilon_{2}} \times\right. \\
\left.\left.\times \sinh \left(\varepsilon_{2} / T\right)-\frac{\cosh \left(\varepsilon_{2} / 2 T\right)}{2}\right]\right) .
\end{gathered}
$$

We can also calculate the projection of the average magnetic moment along $x$ direction. For this purpose we

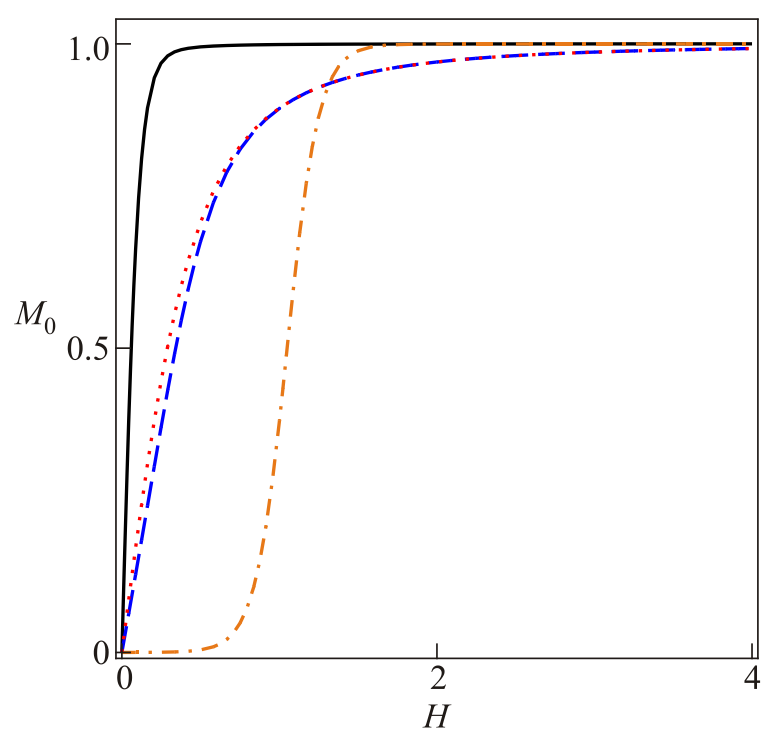

Fig. 1. (Color online) Calculated magnetic field dependences of the projections of the magnetic moment $M_{0}$ for $S=1$ at $T=0.1$. For the "easy axis" case $D=1$, with the small biaxial anizotropy $E=-0.1$ the solid black line shows the field is directed along $z$ axis; the dashed blue line shows the field is directed along $x$ axis. For the "easy-plane" case $D=-1$, with the small in-plane anizotropy $E=-0.1$ the dashed-dotted orange line shows the field is directed along $z$ axis; the dotted red line shows the field is directed along $x$ axis.

replace $x \leftrightarrow z$ in the Hamiltonian $\mathcal{H}$. Hence, the answers for the quantum mechanical averaged value of the $x$ projection of the magnetic moment $M_{x}$ can be obtained by the formal replacement $D \leftrightarrow E$ in Eqs. (10) and (12).

Figures 1 and 2 manifest the magnetic field dependences of the $z$ - and $x$-projections of the magnetic moments for the "easy axis" $D=-1$ and "easy-plane" paramagnet with

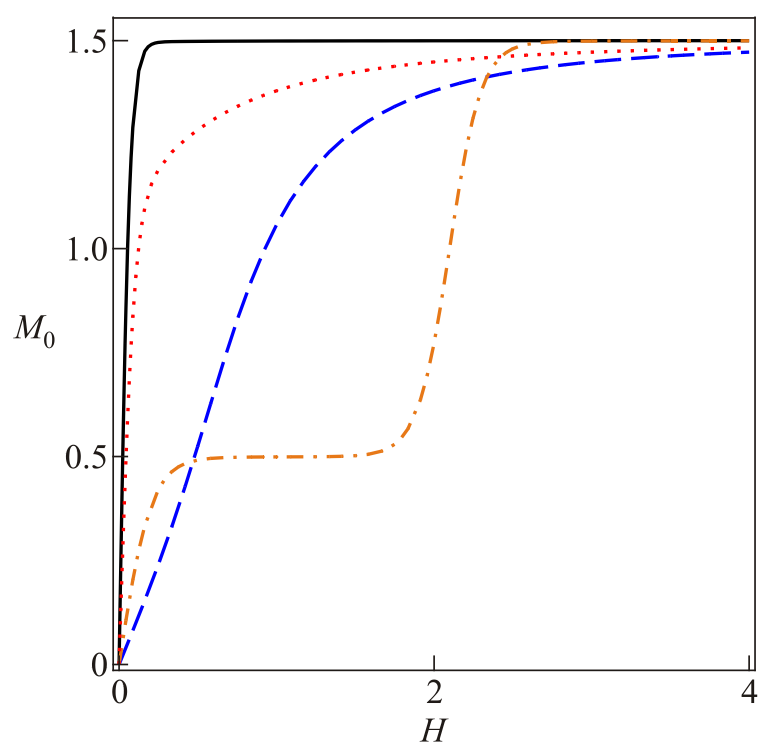

Fig. 2. (Color online) The same as in Fig. 1 but for $S=3 / 2$. The parameters and the notations are the same as in Fig. 1. 
the weak biaxial magnetic anisotropy $E=-0.1$ at low temperature $T=0.1$ for $S=1$ and $3 / 2$, respectively. We see that for different directions of the field the magnetic field behavior manifests different features. We point out that the change of the sign of the weak biaxial anisotropy does not produce essential changes in the magnetic field behavior. At higher temperatures (of order of the maximal value of the magnetic anisotropy) all those features are "smeared out".

\section{Dynamics after the quantum quench}

Now consider the following situation. Suppose at $t=0$ we change the value of the magnetic field from $H_{i}$ (valid at $t \leq 0$ ) to $H_{f}$ (valid for $t>0$ ), known as the quantum quench. Dynamics of any quantum system can be describeed in two ways. In the first way one considers the time evolution of the considered operator (using the Heisenberg equations), and then average the obtained time-dependent value of the operator with respect to the wave function (for the pure state), or the density matrix (for the mixed state). The other way, is to find the time evolution of the wave function (using the Schrödinger equation) or the density matrix (using the Liouville equation), and then average the considered operator with the obtained time-dependent wave function or the density matrix. In the case of exact calculations both ways yield the same answer.

To describe dynamics of the studied spin system under the action of the linearly polarized ac magnetic field let us use, for instance, the first approach. The Liouville equation for density matrix $\rho$ has the form $i \dot{\rho}=[\mathcal{H}, \rho]$, where [.,.] denotes the commutator. Such a behavior is characteristic for a closed system. However, as a rule, the spin system is not isolated. For example, there are processes, which take the energy from the system, i.e., relaxation processes. The relaxation can be considered in a number of ways. The reason for the relaxation of the density matrix is the interaction of the considered system with some environment; such an interaction takes the energy from the system, i.e., our considered system is the open one. For example, for the studied quantum spin system the lattice (i.e., the elastic subsystem of the crystal) can serve as such an environment.

Dynamics of the density matrix of our open system for general Markovian processes is described by the Lindblad master equation [14] (here we write it in the diagonal form)

$$
i \dot{\rho}=[\mathcal{H}, \rho]+i \sum_{j=1}^{N^{2}-1} \gamma_{j}\left(\mathcal{L}_{j} \rho \mathcal{L}_{j}^{\dagger}+\frac{1}{2}\left\{\mathcal{L}_{j}^{\dagger} \mathcal{L}_{j}, \rho\right\}\right)
$$

where $N$ is the dimension of the system, $\{.,$.$\} denotes the$ anticommutator, and the orthonormal and traceless operators $\mathcal{L}_{j}$ are the Lindblad (jump) operators. For $\gamma_{j}=0$ the Lindblad equation is, obviously, the Liouville equation. In the model of random collisions [15] one can write the Lindblad operators as $\mathcal{L}_{j}=\sqrt{\left(\rho_{0}\right)_{j j}}|j\rangle\left\langle j^{\prime}\right|$, and suppose that all $\gamma_{j}$ are equal, which yields

$$
i \dot{\rho}=[\mathcal{H}, \rho]+i \gamma\left(\rho_{0}-\rho\right) .
$$

This form of the master equation was first suggested by Karplus and Schwinger [16]. It was used to describe the relaxation processes of quantum systems under the action of the ac electromagnetic field. It describes the interaction of the considered system with the bath, with the relaxation of the density matrix to $\rho_{0}$ in the steady state. It is useful to substitute $\rho^{\prime}=\exp (\gamma t) \rho$; we obtain $i \dot{\rho}^{\prime}=\left[\mathcal{H}, \rho^{\prime}\right]+\gamma \exp (\gamma t) \rho_{0}$. The used approximation implies equal relaxation times for all eigenmodes of the system. It is equivalent to the Bloch form of relaxation in the theory of the nuclear magnetic resonance [17]. Two relaxation times as in the Bloch approach can be easily introduced in the above scheme by using different relaxation rates for diagonal and non-diagonal components of the density matrix. One can also generalize the approach using, e.g., Torrey's phenomenological theory [18], which adds diffusion processes to the Bloch equations. It is possible to show that the effect of the linear relaxation in the Bloch form is similar to the effect of the relaxation in the Landau-Lifshitz form for magnetic systems [19]. Here we are interested mostly in the homogeneous response, and can neglect the spatial dependence of relaxation.

\section{Closed system}

The response to the quantum quench is strictly nonlinear for the studied model. After some algebra similar to the one, developed in Ref. 20 we obtain for $\gamma=0$ for the closed system for $S=1$

$$
\begin{aligned}
M_{z}(t)=M_{z, i}+ & \frac{2 \sinh (\varepsilon / T)}{2 \cosh (\varepsilon / T)+\exp [(2 D-E) / 2 T]} \times \\
& \times \frac{E^{2} H_{f}}{2 \varepsilon_{i} \varepsilon_{f}^{2}} \sin ^{2}\left(\varepsilon_{f} t\right)
\end{aligned}
$$

where we use units in which $\hbar=1$, and $\varepsilon_{i, f}=\varepsilon\left(H=H_{i, f}\right)$. We see that the average magnetic moment oscillates with time and with the magnitude $H_{f}$ of the quantum quench. For instance, Fig. 3 shows the oscillations of the average value of the magnetic moment along $z$ direction for $S=1$ "easy axis"-like case with $D=-1$ and $E=-0.1$ at $T=0.1$ for $H_{i}=0$.

For $S=3 / 2$ the calculated dependence is

$$
\begin{aligned}
& M_{z}(t)=M_{z, i}+\left[\exp \left(H_{i} / 2 T\right) \cosh \left(\varepsilon_{1 i} / T\right)+\right. \\
& \left.+\exp \left(-H_{i} / 2 T\right) \cosh \left(\varepsilon_{2 i} / 2 T\right)\right]^{-1} \frac{3 H_{f} E^{2}}{2} \times
\end{aligned}
$$




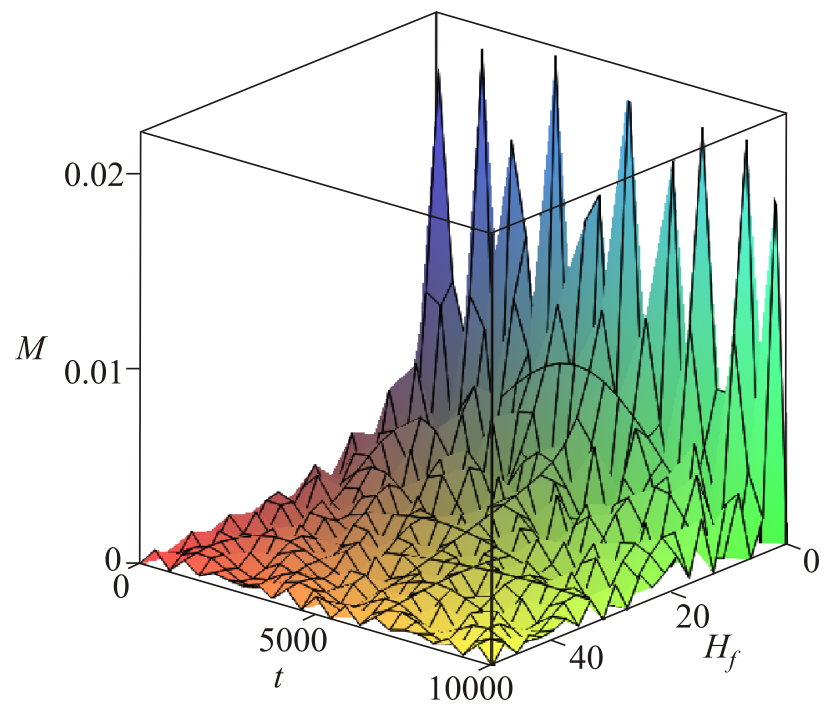

Fig. 3. (Color online) Calculated magnetic field dependence of the $z$-projection of the magnetic moment $M$ on the magnitude of the quantum quench $H_{f}$ and time $t$ at $H_{i}=0$ for the closed $S=1$ "easy axis"-like system $(D=-1, E=-0.1)$ at $T=0.1$.

$$
\begin{gathered}
\times\left(\operatorname { e x p } ( H _ { i } / 2 T ) \frac { 1 } { \varepsilon _ { 1 i } \varepsilon _ { 1 f } ^ { 2 } } \operatorname { s i n } ^ { 2 } ( \varepsilon _ { 1 f } t ) \left[\sinh \left(\varepsilon_{1 i} / T\right) \times\right.\right. \\
\left.\times \frac{\left(H_{i}-D+E / 2\right)}{\varepsilon_{1 i}}+\frac{\cosh \left(\varepsilon_{1 i} / 2 T\right)}{2}\right]- \\
-\exp \left(-H_{i} / 2 T\right) \frac{1}{\varepsilon_{2 i} \varepsilon_{2 f}^{2}} \sin ^{2}\left(\varepsilon_{2 f} t\right)\left[\sinh \left(\varepsilon_{2 i} / T\right) \times\right. \\
\left.\left.\times \frac{\left(H_{i}+D-E / 2\right)}{\varepsilon_{2 i}}-\frac{\cosh \left(\varepsilon_{2 i} / 2 T\right)}{2}\right]\right)
\end{gathered}
$$

where $\varepsilon_{1,2 i, f}=\varepsilon_{1,2}\left(H=H_{i, f}\right)$. Notice that for $S=3 / 2$ we observe the interference of oscillations with two frequences, $\varepsilon_{1,2 f}$, unlike the case for $S=1$, where the magnetic moment oscillates with only one frequency $\varepsilon_{f}$. Figure 4 shows the example of oscillations of the average value of the magnetic moment along $x$ direction for $S=3$ / 2 "easy-plane"-like case with $E=1$ and $D=-0.1$ at $T=0.1$ for $H_{i}=0$.

We see that the magnitude of the oscillations of the $x$-projection is larger than the one for the $z$-projection of the magnetic moment. Notice that for large values of $H_{f}$ the magnitude of oscillations decay, see Figs. 3 and 4.

It is possible to calculate the average in time value, about which the quantum mechanical magnetic moment oscillates after the quantum quench. For $S=1$ we get

$$
\langle M\rangle_{z}=M_{z, i}+\frac{2 \sinh (\varepsilon / T)}{2 \cosh (\varepsilon / T)+\exp [(2 D-E) / 2 T]} \frac{E^{2} H_{f}}{4 \varepsilon_{i} \varepsilon_{f}^{2}}
$$

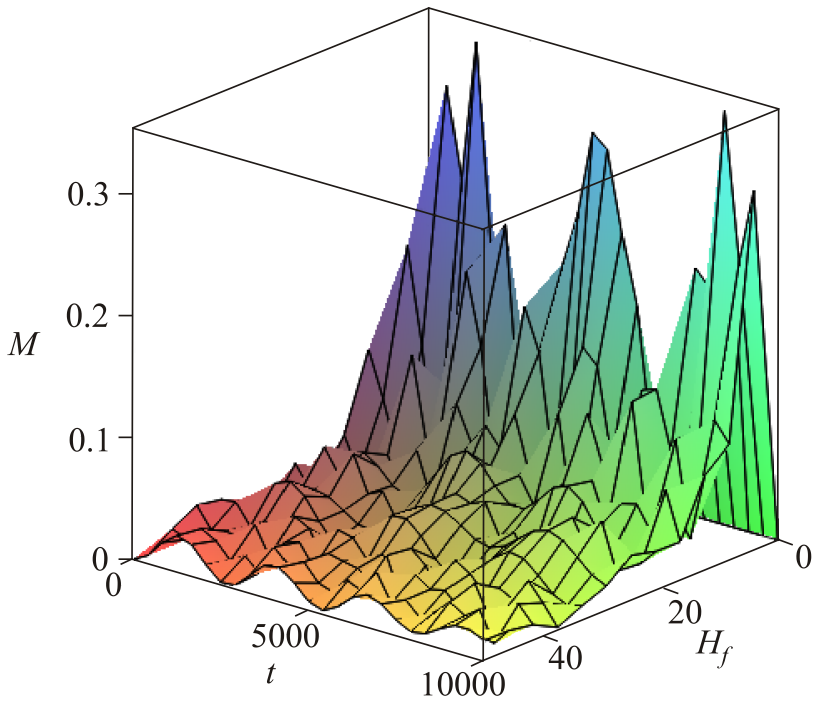

Fig. 4. (Color online) Calculated magnetic field dependence of the $x$-projection of the magnetic moment $M$ on the magnitude of the quantum quench $H_{f}$ and time $t$ at $H_{i}=0$ for the closed $S=3 / 2$ "easy-plane"-like system $(D=-0.1, E=1)$ at $T=0.1$.

and for $S=3 / 2$ we obtain

$$
\begin{gathered}
\langle M\rangle_{z}=M_{z, i}+\left[\exp \left(H_{i} / 2 T\right) \cosh \left(\varepsilon_{1 i} / T\right)+\right. \\
\left.+\exp \left(-H_{i} / 2 T\right) \cosh \left(\varepsilon_{2 i} / 2 T\right)\right]^{-1} \frac{3 H_{f} E^{2}}{4} \times \\
\times\left(\operatorname { e x p } ( H _ { i } / 2 T ) \frac { 1 } { \varepsilon _ { 1 i } \varepsilon _ { 1 f } ^ { 2 } } \left[\sinh \left(\varepsilon_{1 i} / T\right) \times\right.\right. \\
\left.\times \frac{\left(H_{i}-D+E / 2\right)}{\varepsilon_{1 i}}+\frac{\cosh \left(\varepsilon_{1 i} / 2 T\right)}{2}\right]- \\
-\exp \left(-H_{i} / 2 T\right) \frac{1}{\varepsilon_{2 i} \varepsilon_{2 f}^{2}}\left[\sinh \left(\varepsilon_{2 i} / T\right) \times\right. \\
\left.\left.\times \frac{\left(H_{i}+D-E / 2\right)}{\varepsilon_{2 i}}-\frac{\cosh \left(\varepsilon_{2 i} / 2 T\right)}{2}\right]\right) .
\end{gathered}
$$

Equations (15)-(18) describe totally dynamical behavior for the closed system.

Figures 5 and 6 show the dependences of the average in time values, about which magnetic moments oscillate on the values of the initial $H_{i}$ and final $H_{f}$ values of the magnetic field at $T=0.1$. In Fig. 5 the $S=1$ case is shown for the "easy axis"-like case $(D=-1$ and $E=-0.1)$ for the $x$-projection.

In Fig. 6 the $S=3 / 2$ case is shown for the "easyplane"-like case ( $D=1$ and $E=-0.1)$ for the $z$-projection.

These results show that the average value of the magnetic moment is mostly determined by the initial value of the magnetic field $H_{i}$, and the final value $H_{f}$ plays an essential role if it has the sign, different from the sign of $H_{i}$, and for $H_{i}=0$. 


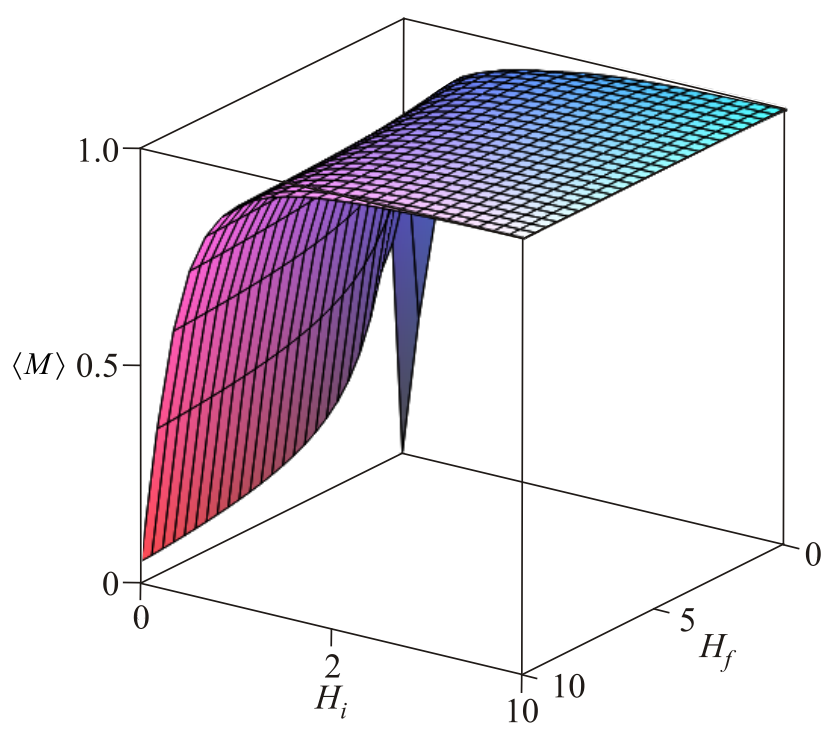

Fig. 5. (Color online) Calculated dependences of the $x$-projection of the average in time value of the magnetic moment $M$ on the magnitude of the quantum quench $H_{f}$ and the initial value of the field $H_{i}$ for the closed $S=1$ "easy axis"-like system $(D=-1$, $E=-0.1)$ at $T=0.1$.

\section{Open system}

If the considered system is the part of the larger subsystem (the bath), i.e., we deal with the open system case, we need to take into account the relaxation processes due to the exchange of the energy between the bath and our subsystem, see above. For example, we can use the KarplusSchwinger form of the Lindblad master equation for the density matrix, where the linear relaxation $\gamma$ is introduced. The result is equivalent to the standard Bloch approach to the equations of motion for spin projections. Notice that the inclusion of the linear relaxation implies multiplication of the time-dependent parts of Eqs. (15) and (16) by the multiplier $\exp (-\gamma t)$, and those equations are valid for $t<\gamma^{-1}$ in the dynamical regime. In the steady-state regime $t \gg \gamma^{-1}$ after some calculations we obtain for spin $S=1$

$$
\begin{aligned}
M_{z}^{\text {st }}=M_{z, i}+\frac{2 \sinh (\varepsilon / T)}{2 \cosh (\varepsilon / T)+\exp [(2 D-E) / 2 T]} \times \\
\times \frac{E^{2} H_{f}}{2 \varepsilon_{i}\left(\varepsilon_{f}^{2}+\gamma^{2}\right)} .
\end{aligned}
$$

Here we have supposed that the system decays to the state with $H=H_{i}$.

The dependence of the steady-state magnetic moment $M$ on the magnitude of the quantum quench $H_{f}$ for $H_{i}=0$ (i.e., the switching on the field) for $S=1$ for two directions of the magnetic field and for the "easy axis" and the "easy-plane" cases (with the weak biaxial magnetic anisotropy) is shown in Fig. 7.

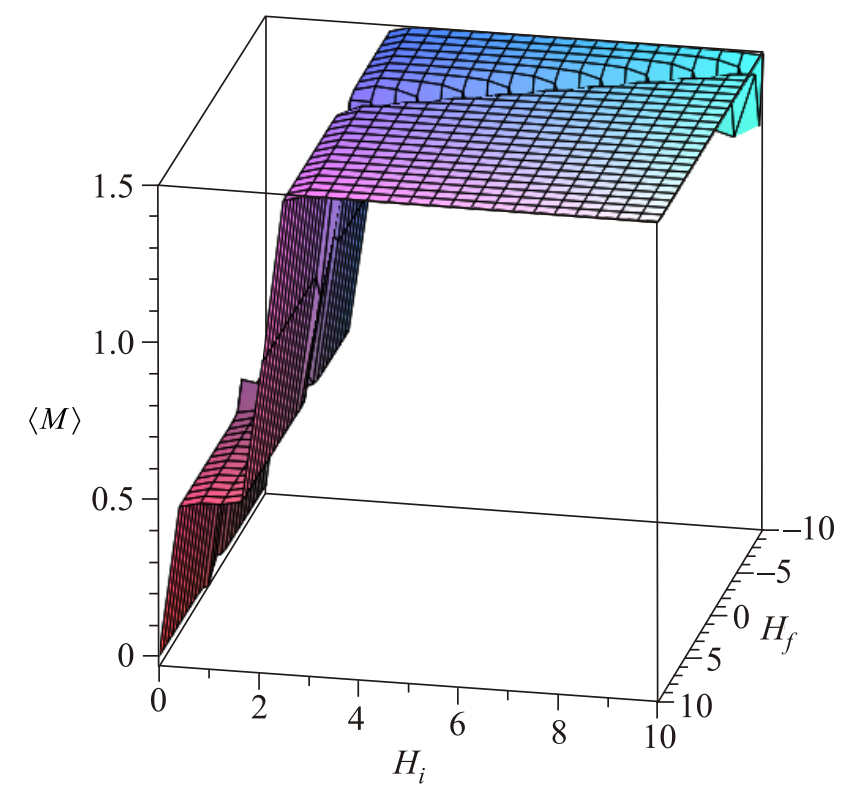

Fig. 6. (Color online) Calculated dependences of the $z$-projection of the average in time value of the magnetic moment $M$ on the magnitude of the quantum quench $H_{f}$ and the initial value of the field $H_{i}$ for the closed $S=3 / 2$ "easy-plane"-like system $(D=1$, $E=-0.1)$ at $T=0.1$.

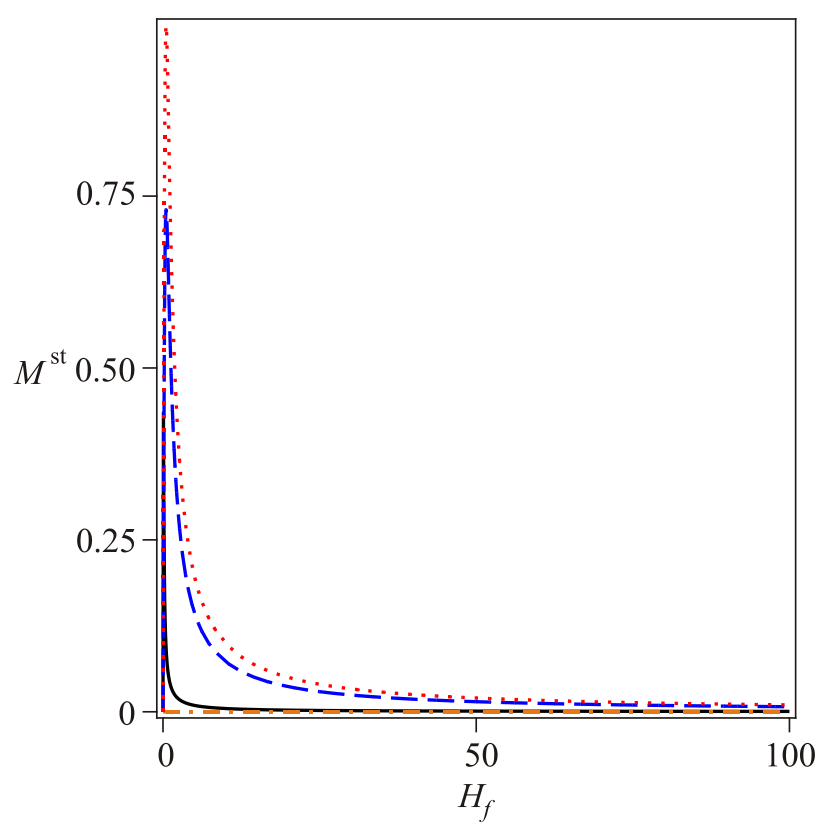

Fig. 7. (Color online) Calculated magnetic field dependences of the projections of the magnetic moment $M$ on the magnitude of the quantum quench $H_{f}$ at $H_{i}=0$ (switching on the field) in the steady-state regime for $S=1$ at $T=0.1$ with $\gamma=0.01$. For the "easy axis" case $D=1$, with the small biaxial anizotropy $E=-0.1$ the solid black line shows the field is directed along $z$ axis; the dashed blue line shows the field is directed along $x$ axis. For the "easy-plane" case $D=1$, with the small in-plane anizotropy $E=-0.1$ the dashed-dotted orange line shows the field is directed along $z$ axis; the dotted red line shows the field is directed along $x$ axis. 
We see that the average magnetic moment decays in the steady-state regime with the magnitude of the quantum quench. The oscillations, characteristic for the dynamical regime and the closed system, are exhausted.

For the case $S=3$ / 2 we get

$$
\begin{gathered}
M_{z}^{\text {st }}=M_{z, i}+\left[\exp \left(H_{f} / 2 T\right) \cosh \left(\varepsilon_{1 f} / T\right)+\right. \\
\left.+\exp \left(-H_{f} / 2 T\right) \cosh \left(\varepsilon_{2 f} / 2 T\right)\right]^{-1} \frac{3 H_{f} E^{2}}{2} \times \\
\times\left(\operatorname { e x p } ( H _ { f } / 2 T ) \frac { 1 } { \varepsilon _ { 1 i } ( \varepsilon _ { 1 f } ^ { 2 } + \gamma ^ { 2 } ) } \left[\sinh \left(\varepsilon_{1 f} / T\right) \times\right.\right. \\
\left.\times \frac{\left(H_{f}-D+E / 2\right)}{\varepsilon_{1 f}}+\frac{\cosh \left(\varepsilon_{1 f} / 2 T\right)}{2}\right]- \\
-\exp \left(-H_{f} / 2 T\right) \frac{1}{\varepsilon_{2 i}\left(\varepsilon_{2 f}^{2}+\gamma^{2}\right)}\left[\sinh \left(\varepsilon_{2 f} / T\right) \times\right. \\
\left.\left.\times \frac{\left(H_{f}+D-E / 2\right)}{\varepsilon_{2 f}}-\frac{\cosh \left(\varepsilon_{2 f} / 2 T\right)}{2}\right]\right) .
\end{gathered}
$$

Figure 8 shows the dependence on the magnitude of the quantum quench $H_{f}$ of the steady-state value of the average magnetic moment for $S=3 / 2$ after switching the magnetic field from $H_{i}=0$ for the "easy axis" and the "easy-plane" anisotropy (with the weak biaxial anisotropy) for $z$ and $x$ directions of the magnetic field. The parameters and the notations are the same as in Fig. 7. The average magnetic moment decays in the steady-state regime with the magnitude of the quantum quench as for $S=1$. However, the value of the average magnetic moment in the steady-state regime for $S=3 / 2$ can become negative.

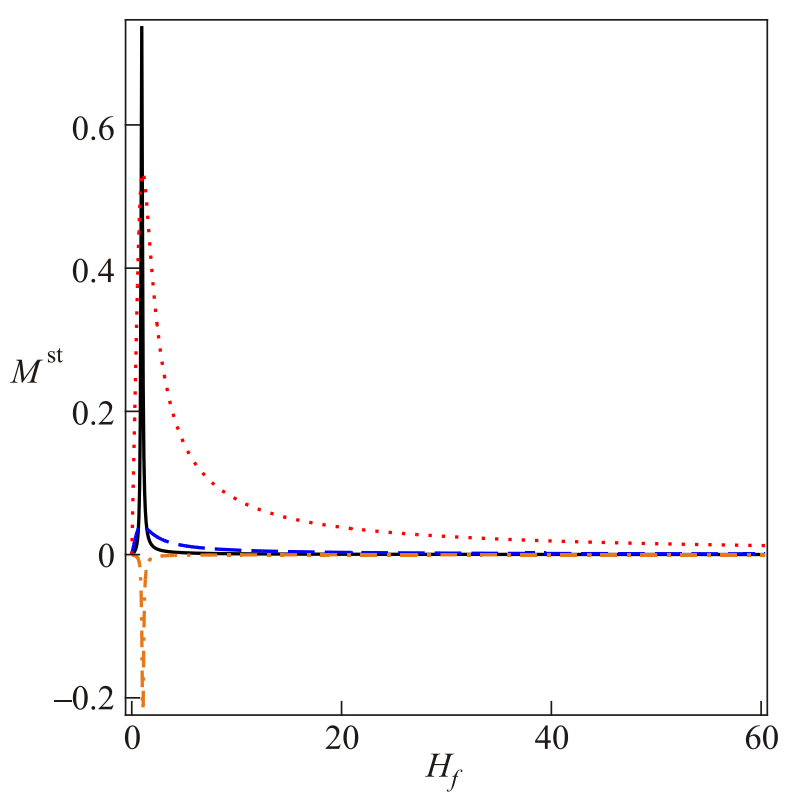

Fig. 8. (Color online) The same as in Fig. 7 but for $S=3 / 2$. The parameters and the notations are the same as in Fig. 7.

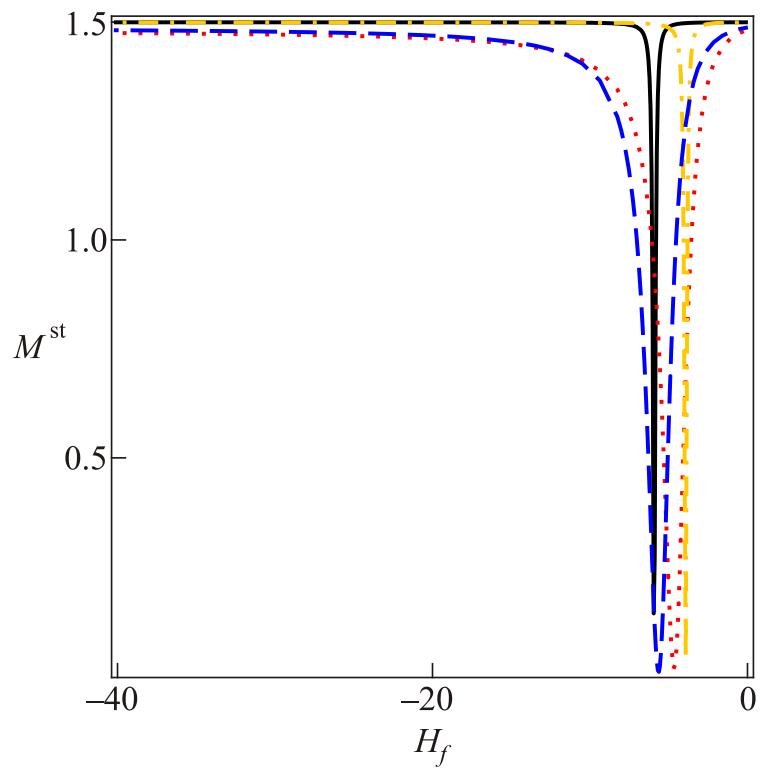

Fig. 9. (Color online) Calculated magnetic field dependences of the projections of the magnetic moment $M$ on the magnitude of the quantum quench $H_{f}$ starting from $H_{i}=5$ (switching off the field) in the steady-state regime for $S=3 / 2$ at $T=0.1$ with $\gamma=0.01$. The parameters and the notations are the same as in Fig. 7.

On the other hand, for switching off the field from $H_{i}=5$ the dependence of the steady-state average magnetic moment on the magnitude of the quantum quench $H_{f}$ is shown in Fig. 9 for $S=3 / 2$. The parameters and the notations are the same as in Fig. 7. It turns out that the average magnetic moment goes to zero not at $H_{f}=-H_{i}$ as it is naively expected; zero value of the average magnetic moment is determined by the values of the magnetic anisotropy constants. Then, at large negative values of $H_{f}$, the

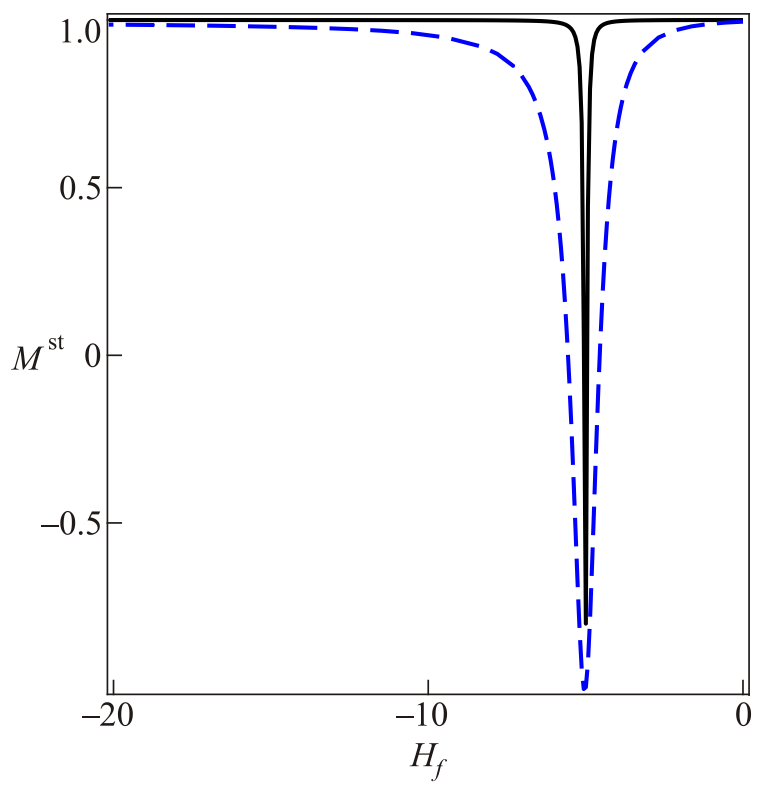

Fig. 10. (Color online) The same as in Fig. 9 but for $S=1$. The parameters and the notations are the same as in Fig. 7. 
average magnetic moment gets the value determined by $H=H_{i}$.

For $S=1$ the dependences for switching off the field are shown in Fig. 10. Notice that the dependences are similar for $D= \pm 1$. It turns out that for $S=1$ the steady-state magnetic moment for $H_{f}=-H_{i}$ when switching off the field becomes negative (unlike the case $S=3 / 2$ ). Also, unlike $S=3 / 2$ the steady-state magnetic moment is minimal at $H_{f}=-H_{i}$. Then, for large negative values of $H_{f}$, it gets the value determined by $H=H_{i}$, as for $S=3 / 2$.

Those effects are the manifestation of the specific hysteresis phenomenon, which is known to be totally dynamical in single molecular magnets [21].

\section{Summary}

In summary, we have studied dynamical non-equilibrium effects in a quantum spin system, which permits exact analytical solution in both closed and open cases, i.e., if the system is isolated, or it is connected to the bath. The exact analytic results are obtained for the bi-axial paramagnet, both for the "easy axis"- and the "easy-plane"-like situations, and for the field directed along both principal axes of the system.

We have shown that quantum quench of the external magnetic field produces a nonlinear response. Namely, for the closed system the average magnetic moment oscillates with time and with the final value of the external field. The value of the magnetic moment, around which oscillations persist, is mostly determined by the initial value of the field. Such oscillations exist also for the open system, connected to the thermostat, in the dynamical regime. For the steady-state regime in the open case the oscillations are "smeared out". We have shown that such dynamical effects produce specific hysteresis phenomena in the considered single spin system.

1. A. Polkovnikov, K. Sengupta, A. Silva, and M. Vengalattore, Rev. Mod. Phys. 83, 863 (2011); A.A. Zvyagin, Fiz. Nizk. Temp. 42, 1240 (2016) [Low Temp. Phys. 42, 971 (2016)].

2. M.A. Nielsen and I. Chuang, Quantum Computation and Quantum Information, Cambridge University Press, Cambridge (2000).

3. I. Bloch, J. Dalibard, and W. Zwerger, Rev. Mod. Phys. 80, 885 (2008); T. Kinoshita, T. Wenger, and D.S. Weiss, Nature (London) 440, 900 (2006); S. Trotzky, Y.-A. Chen, A. Flesch, I.P. McCulloch, U. Schollwock, J. Eisert, and I. Bloch, Nat. Phys. 8, 325 (2012); M. Gring, M. Kuhnert, T. Langen, T. Kitagawa, B. Rauer, M. Schreitl, I. Mazets, D.A. Smith, E. Demler, and J. Schmiedmayer, Science 337, 1318 (2012); T. Fukuhara, P. Schauss, M. Endres, S. Hild, M. Cheneau, I. Bloch, and C. Gross, Nature 502, 76 (2013).

4. B. Ferguson and X.-C. Zhang, Nature Mater. 1, 26 (2002); M. Tonouchi, Nature Photon. 1, 97 (2007); T. Kampfrath, A. Sell, G. Klatt, A. Pashkin, S. Mährlein, T. Dekorsy, M. Wolf,
M. Fiebig, A. Leitenstorfer, and R. Huber, Nature Photon. 5, 31 (2011).

5. B.E. Cole, J.B. Williams, B.T. King, M.S. Sherwin, and C.R. Stanley, Nature 410, 60 (2001); R. Huber, F. Tauser, A. Brodschelm, M. Bichler, G. Abstreiter, and A. Leitenstorfer, Nature 414, 286 (2001); R.A. Kaindl, M.A. Carnahan, D. Hägele, R. Lövenich, and D.S. Chemla, Nature 423, 734 (2003); S. Iwai, M. Ono, A. Maeda, H. Matsuzaki, H. Kishida, H. Okamoto, and Y. Tokura, Phys. Rev. Lett. 91, 057401 (2003); S.G. Carter, V. Birkedal, C.S. Wang, L.A. Coldren, A.V. Maslov, D.S. Citrin, and M.S. Sherwin, Science 310, 651 (2005); L. Perfetti, P.A. Loukakos, M. Lisowski, U. Bovensiepen, H. Berger, S. Biermann, P.S. Cornaglia, A. Georges, and M. Wolf, Phys. Rev. Lett. 97, 067402 (2006); J. Kröll, J. Darmo, S.S. Dhillon, X. Marcadet, M. Calligaro, C. Sirtori, and K. Unterrainer, Nature 449, 698 (2007); J.R. Danielson, Y.-S. Lee, J.P. Prineas, J.T. Steiner, M. Kira, and S.W. Koch, Phys. Rev. Lett. 99, 237401 (2007); S. Wall, D. Brida, S.R. Clark, H.P. Ehrke, D. Jaksch, A. Ardavan, S. Bonora, H. Uemura, Y. Takahashi, T. Hasegawa, H. Okamoto, G. Cerullo, and A. Cavalleri, Nat. Phys. 7, 114 (2011).

6. S. Zherlitsyn, B. Wustmann, T. Herrmannsdörfer, and J. Wosnitza, IEEE Trans. Appl. Supercond. 22, 3 (2012); F. Weickert, B. Meier, S. Zherlitsyn, T. Herrmannsdörfer, R. Daou, M. Nicklas, J. Haase, F. Steglich, and J. Wosnitza, Meas. Sci. Technol. 23, 105001 (2012).

7. For the review use B.A. Ivanov, Fiz. Nizk. Temp. 40, 119 (2014) [Low Temp. Phys. 40, 91 (2014)].

8. J.P. Gauyacq and N. Lorente, Surf. Science 630, 325 (2014); J.P. Gauyacq and N. Lorente, Phys. Rev. B 87, 195402 (2013).

9. E.G. Galkina, V.I. Butrim, Yu.A. Fridman, B.A. Ivanov, and F. Nori, Phys. Rev. B 88, 144420 (2013); E.G. Galkina, B.A. Ivanov, and V.I. Butrim, Fiz. Nizk. Temp. 40, 817 (2014) [Low Temp. Phys. 40, 635 (2014)].

10. V.M. Loktev and V.S. Ostrovskii, Fiz. Nizk. Temp. 20, 983 (1994) [Low Temp. Phys. 20, 775 (1994)].

11. S. Sanvito and A.R. Rocha, J. Comput. Theor. Nanosci. 3, 624 (2006); G. Christou, D. Gatteschi, D.N. Hendrickson, and R. Sessoli, Mater. Res. Soc. Bull. 25, 66 (2000).

12. J.R. Friedman, M.P. Sarachik, J. Tejada, and R. Ziolo, Phys. Rev. Lett. 76, 3830 (1996); W. Wernsdorfer and R. Sessoli, Science 284, 133 (1999); D. Gatteschi, R. Sessoli, and J. Villain, Molecular Nanomagnets, Oxford University Press, New York (2007); N. Ishikawa, M. Sugita, T. Ishikawa, S. Koshihara, and Y. Kaizu, J. Am. Chem. Soc. 125, 8694 (2003); M.S. Alam, V. Dremov, P. Müller, A.V. Postnikov, S.S. Mal, F. Hussain, and U. Kortz, Inorg. Chem. 45, 2866 (2006); M.A. AlDamen, J.M. Clemente-Juan, E. Coronado, C. Marti-Gastaldo, and A. Gaita-Arino, J. Am. Chem. Soc. 130, 8874 (2008); J.M. Zadrozny, D.J. Xiao, M. Atanasov, G.J. Long, F. Grandjean, F. Neese, and J.R. Long, Nat. Chem. 5, 577 (2013); P.E. Kazin, M.A. Zykin, W. Schnelle, C. Felser, and M. Jansen, Chem. Commun. 50, 9325 (2014); J. Liu, Y.-C. Chen, J.-L. Liu, V. Vieru, L. Ungur, J.-H. Jia, 
L.F. Chibotaru, Y. Lan, W. Wernsdorfer, S. Gao, X.-M. Chen, and M.-L. Tong, J. Am. Chem. Soc. 138, 5441 (2016).

13. T. Satoh, R. Iida, T. Higuchi, Y. Fujii, A. Koreeda, H. Ueda, T. Shimura, K. Kuroda, V.I. Butrim, and B.A. Ivanov, Nat. Commun. 8, 638 (2017).

14. G. Lindblad, Commun. Math. Phys. 48, 119 (1976); V. Gorini, A. Kossakovski, and E.C.G. Sudarshan, J. Math. Phys. 17, 821 (1976).

15. Y.A. Ilyinskiy and L.V. Keldysh, Interaction of the Electromagnetic Radiation with the Matter, Moscow State University Publishers, Moscow (1989) (in Russian).

16. R. Karplus and J. Schwinger, Phys. Rev. 73, 1020 (1948).

17. F. Bloch, Phys. Rev. 70, 460 (1946).

18. H.C. Torrey, Phys. Rev. 104, 563 (1956).

19. A.A. Zvyagin, Fiz. Nizk. Temp. 41, 938 (2015) [Low Temp. Phys. 41, 730 (2015)].

20. A.A. Zvyagin, Phys. Rev. B 92, 184507 (2015).

21. A. Caneschi, D. Gatteschi, C. Sangregorio, R. Sessoli, L. Sorace, A. Cornia, M.A. Novak, C. Paulsen, and W. Wernsdorfer, J. Magn. Magn. Mater. 200, 182 (1999); M. Thorwart, P. Reimann, P. Jung, and R.F. Fox, Phys. Lett. A 239, 233 (1998); T. Kawakami, H. Nitta, M. Takahata, M. Shoji, Y. Kitagawa, M. Nakano, M. Okumura, and K. Yamaguchi, Polyhedron 28, 2092 (2009).

\section{Квантове гартування двовісних квантових спінових систем}

\begin{abstract}
А.А. Звягін
Досліджено динамічні нерівноважні ефекти після квантового гартування в квантових спінових системах, які допускають точні аналітичні рішення як в закритому, так і у відкритому випадках. Точні аналітичні рішення отримані для двовісного парамагнетика як в легкоплощинних, так і легковісній ситуаціях і для поля, прикладеного вздовж двох голо-
\end{abstract}

вних осей системи. Квантове гартування зовнішнім магнітним полем призводить до нелінійного відгуку. Для закритої системи середній магнітний момент осцилюе із часом та 3 фінальним значенням зовнішнього поля. Такі осциляції існують також і у відкритій системі, що контактує з термостатом, в динамічному режимі. Для сталого режиму у відкритій системі осциляції пригнічені. Нерівноважні ефекти створюють специфічні гістерезисні явища в даній моноспіновій системі.

Ключові слова: квантове гартування, двовісна магнітна анізотропія, динамічний гістерезис.

\section{Квантовая закалка двуосных квантовых спиновых систем}

\section{А.А. Звягин}

Исследованы динамические неравновесные эффекты после квантовой закалки в квантовых спиновых системах, которые допускают точные аналитические решения как в закрытом, так и в открытом случаях. Точные аналитические решения получены для двуосного парамагнетика как в легкоплоскостной, так и легкоосной ситуациях и для поля, приложенного вдоль двух главных осей системы. Квантовая закалка внешним магнитным полем приводит к нелинейному отклику. Для закрытой системы средний магнитный момент осциллирует со временем и с финальным значением внешнего поля. Такие осцилляции существуют также и в открытой системе, контактирующей с термостатом, в динамическом режиме. Для установившегося режима в открытой системе осцилляции подавлены. Неравновесные эффекты создают специфические гистерезисные явления в рассматриваемой моноспиновой системе.

Ключевые слова: квантовая закалка, двуосная магнитная анизотропия, динамический гистерезис. 